D. Quintanar-Guerrero

E. Allémann

E. Doelker

H. Fessi

\section{A mechanistic study of the formation of polymer nanoparticles by the emulsification-diffusion technique}

\begin{abstract}
propose a mechanism based on the "diffusion-stranding" mechanism for spontaneous emulsification. In this mechanism, the diffusion of solvent causes local supersaturation near the interface, and nanoparticles are formed, due to the phase transformation and polymer aggregation that occur in these regions. This interpretation is supported by the turbidity measurements made at different polymer concentrations and stirring rates.
\end{abstract}

Key words Polymer - nanoparticles emulsification-diffusion method spontaneous emulsification interfacial turbulence - diffusion stranding

\section{Introduction}

During the last two decades, polymeric biodegradable colloidal carriers have shown promise as controlled drug delivery dosage forms for specific sites in the body. In general, biodegradable nanoparticles (nanospheres or nanocapsules) can be prepared by two methods: either by a polymerization of dispersed monomers or from a dispersion of preformed polymers. Extensive investigations have been made on polyalkylcyanoacrylates nanoparticles and their close derivatives, prepared by emulsion-polymerization processes $[1,2]$. Due to the potentially toxic effects of residual substances present after a polymerization reaction, in addition to probable cross-reactions with the drug, nanoencapsulation techniques using preformed, well-defined polymers are preferable $[3,4]$.

Recently, a new method for manufacturing nanospheres from preformed polymers, namely, emulsification-diffusion, has been described $[5,6]$. This technique presents clear advantages over other existing methods, such as (a) the use of pharmaceutically acceptable organic solvents, (b) no need of high-pressure homogenizers or ultrasonication, (c) high yields, (d) high reproducibility; (e) easy to scale up and (f) rapid purification by cross-flow filtration. The process involves the formation of an $\mathrm{o} / \mathrm{w}$ emulsion with a partially water-soluble solvent, which contains the biodegradable polymer, and with an aqueous phase containing a stabilizer such as poly(vinyl alcohol). The subsequent addition of water to the system causes the 
diffusion of the solvent into the external phase, with the subsequent aggregation of the polymer held in the globules to form nanospheres, as a product of the newly generated nonsolvent phase. The mechanism by which the diffusion of solvent from the globules induces polymer aggregation and hence nanosphere formation, has been attributed to the formation of new nanometer-sized globules, during diffusion [6], but the exact mechanism remains unclear. Thus, a better understanding of this phenomenon is important to control and optimize the variables involved. The aim of this work was to study the effect of varying conditions of the emulsification-diffusion process such as polymer/stabilizer ratio, internal phase/dispersion medium ratio, viscosity of the dispersion medium, addition rate of the dilution medium, presence of electrolyte during diffusion and stirring rate, in order to understand better the mechanism of formation of polymeric nanoparticles. Poly(D,L-lactic acid) (PLA) was used as a polymer model. Turbidimetric evaluations during the diffusion stage were carried out, to determine the globule transitions from PLA solution to solid particles.

\section{Materials and methods}

\section{Materials}

PLA (Medisorb® $100 \mathrm{D}, \mathrm{L}$ ) with an average molecular mass of 130550 and an inherent viscosity of $0.72 \mathrm{dlg}^{-1}$ in chloroform was supplied by Medisorb Technologies L.P. (Cincinnati, OH, USA). Propylene carbonate (PC) of analytical grade was chosen as a partially water-soluble solvent (Fluka, Buchs, Switzerland). Poly(vinyl alcohol) (PVAL) with an average molecular mass of 26000 was used as supplied (Mowiol ${ }^{\circledR}$ 4-88, Hoechst, Frankfurt-amMain, Germany). Dichloromethane and acetone of HPLC grade were obtained from Fluka. Distilled water was purified using a Milli-Q system (Millipore, USA-Bedford, M.D.). All other reagents were of analytical grade and used without further purification.

\section{Methods}

\section{Nanoparticle preparation}

Nanoparticles were formed according to the method described previously [6]. PC and water were mutually saturated for $1 \mathrm{~min}$ before use in order to ensure the initial thermodynamic equilibrium of both the liquids. Typically, $200 \mathrm{mg}$ of PLA were dissolved in $10 \mathrm{ml}$ of water-saturated $\mathrm{PC}$ and this organic solution (internal phase) was emulsified with $20 \mathrm{ml}$ of a $5 \% \mathrm{w} / \mathrm{v}$ PVAL PC-saturated aqueous solution (dispersion medium) using a high-speed homogenizer (Ultra-Turrax T 25, IKA Lab-Technik, Germany) at $8000 \mathrm{rpm}$ for $10 \mathrm{~min}$. After formation of an oil-in-water emulsion, $80 \mathrm{ml}$ of water (dilution medium) were added to the system in order to allow diffusion of PC into the continuous phase, thus causing the aggregation of the polymer in nanoparticles (Fig. 1). Some batches were prepared according to the emulsification solvent-evaporation technique using dichloromethane as the polymer solvent [7]. These experiments were conducted in order to compare the particle size obtained by this technique with that from the emulsification diffusion method.

\section{Viscosity measurement}

The viscosity of the PVAL solutions was determined using a rotating viscosimeter (Rheomat $15 \mathrm{~T}-\mathrm{F}$ Contraves, Zürich, Switzerland) at room temperature.

\section{Globule size and nanoparticle size measurement}

The mean globule size and polydispersity (index expressed from 0 to 9) of PC-in-water emulsions prepared before the diffusion step were measured using a Coulter NanoSizer ${ }^{\circledR}$ TM (Coulter Electronics Harpenden, Herfordshire, UK) previously checked with polystyrene latex mean diameter $197 \pm 10 \mathrm{~nm}$ (Duke Scientific Co., Palo Alto USA). To perform the measurements, the emulsions were diluted using the dispersion medium and the size was rapidly measured for $2 \mathrm{~min}$. Nanoparticle size analysis was carried out by dilution of $250 \mu \mathrm{l}$ of the nanoparticle suspension $(\sim 0.2 \% \mathrm{w} / \mathrm{v})$ to $5 \mathrm{ml}$ with filtered distilled water, also using the Nano-Sizer. Measurements were made in triplicate for all the batches.

\section{Scanning electron microscopy (SEM)}

Some batches were purified and concentrated by crossflow filtration (Minitan $\AA$, Millipore, USA-Bedford, MD), as previously described [6]. A sample of the dispersion was finely spread over a slab and dried under vacuum. The sample was shadowed in a cathodic evaporator with a layer of gold ( $\sim 20 \mathrm{~nm}$ thick). Then, the surface was observed using a JSM-6400 scanning electron microscope (JEOL, Tokyo, Japan).

\section{Turbidimetric analysis}

The turbidity measurements were carried out to evaluate globule size changes and polymer aggregation during the 
Fig. 1 Schematic representation of the nanoparticle preparation process

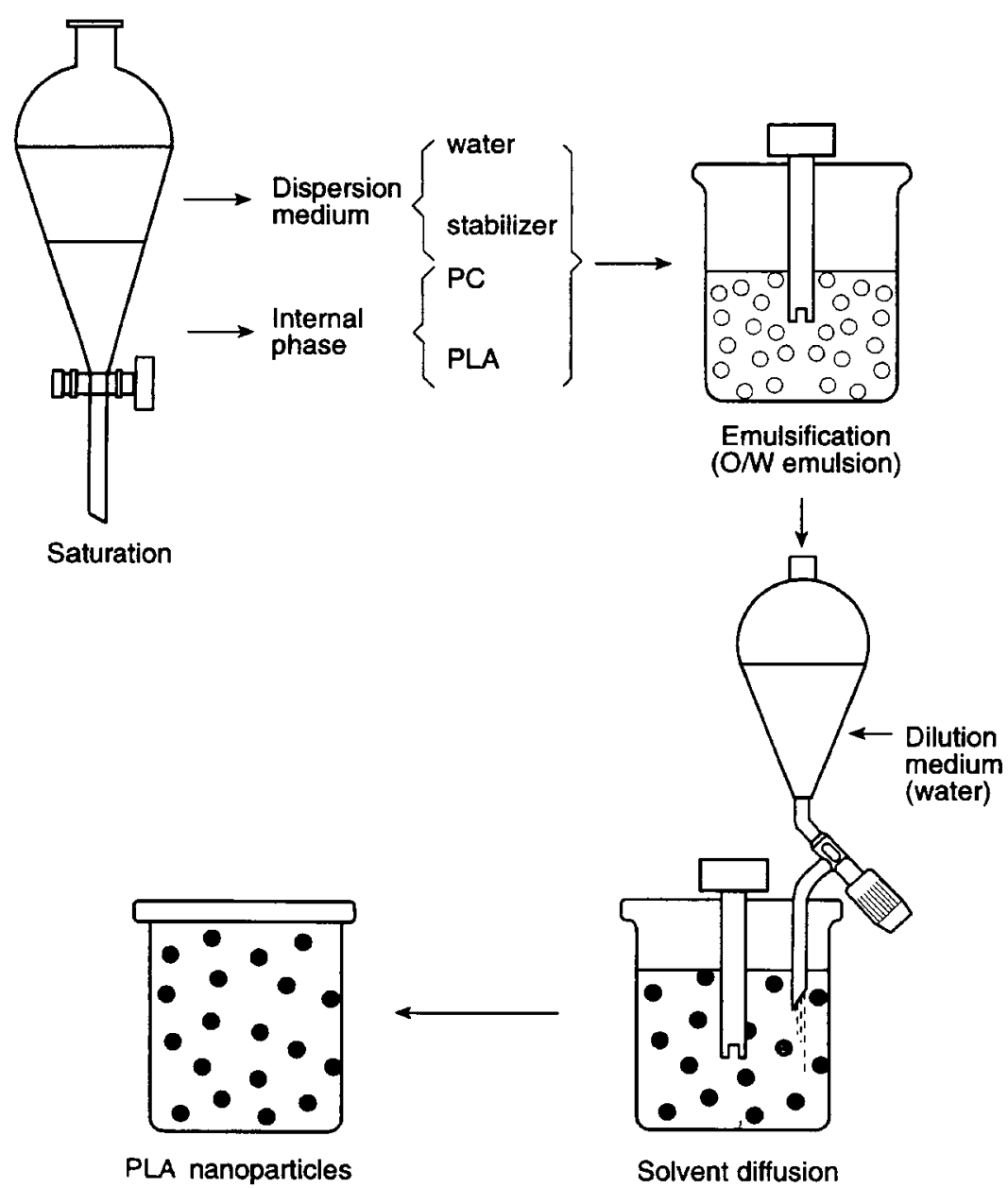

diffusion step. The PC-in-water emulsion was prepared according to the method described above. Typically, the diffusion step was followed by the gradual addition of $5 \mathrm{ml}$ of water at $10 \mathrm{~min}$ intervals, the system being kept at $25 \pm 2{ }^{\circ} \mathrm{C}$ by a water recirculation system. Samples of 50 , 100 or $200 \mu 1$ were taken from the system depending on the PLA concentration and diluted to $5 \mathrm{ml}$ with fresh dispersion medium. The turbidity $(\tau)$ of the samples was assayed spectrophotometrically (Beckman 35, Irvine CA, USA) $10 \mathrm{~min}$ after dilution, at $\lambda=360 \mathrm{~nm}$. The absorbance $(A)$ obtained was related to $\tau$ by the relationship $\tau=2.303 \mathrm{~A} / \mathrm{l}$, where $l$ represents the optical pathlength [8]. The dilution effect due to the added water was taken into account for the calculations. Evaluations were made by changing the PLA concentration of the inner phase as well as the stirring speed using a variable speed stirrer with a stainlesssteel propeller (IKA R 1382, Janke \& Kunke, Staufen, Germany).

\section{Results and discussion}

It is well known that the emulsification of oil in water by mechanical shear produces, in most cases, an emulsion droplet whose size is $2-3 \mu \mathrm{m}$ and down to $1 \mu \mathrm{m}$ in exceptional circumstances [9]. However, if it is assumed that one polymer particle is formed in each emulsion droplet when the solvent is removed, then one can expect a good correlation between the initial droplet size and the final particle size. This supposition seems to be valid in the case of the dichloromethane-in-water emulsion solvent evaporation system. The particle size obtained, under the emulsification conditions used, indicated a diameter larger than $1 \mu \mathrm{m}$, ranging from $1110 \pm 91 \mathrm{~nm}$ for $2 \% \mathrm{w} / \mathrm{v}$ PLA to $1420 \pm 82 \mathrm{~nm}$ for $5 \% \mathrm{w} / \mathrm{v}$ PLA. In contrast, when the particles were formed by the process of emulsification-diffusion, the mean diameter of the resultant spheres 
(Fig. 2) decreased drastically in relation to the initial globule diameter, from $2620 \pm 243 \mathrm{~nm}$ to the submicron range, even at high PLA/PVAL ratios and low internal phase/ dispersion medium ratios (Fig. 3). Furthermore, no correlation was found between the calculated particle volume and the droplet volume, assuming one spherical, solid

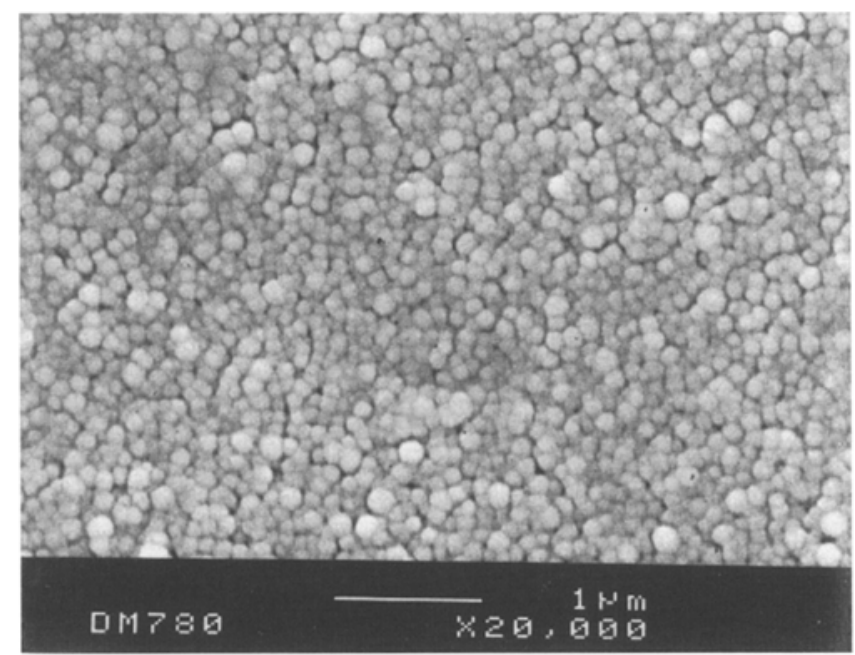

Fig. 2 Scanning electron micrography of PLA nanoparticles prepared by the emulsification-diffusion method

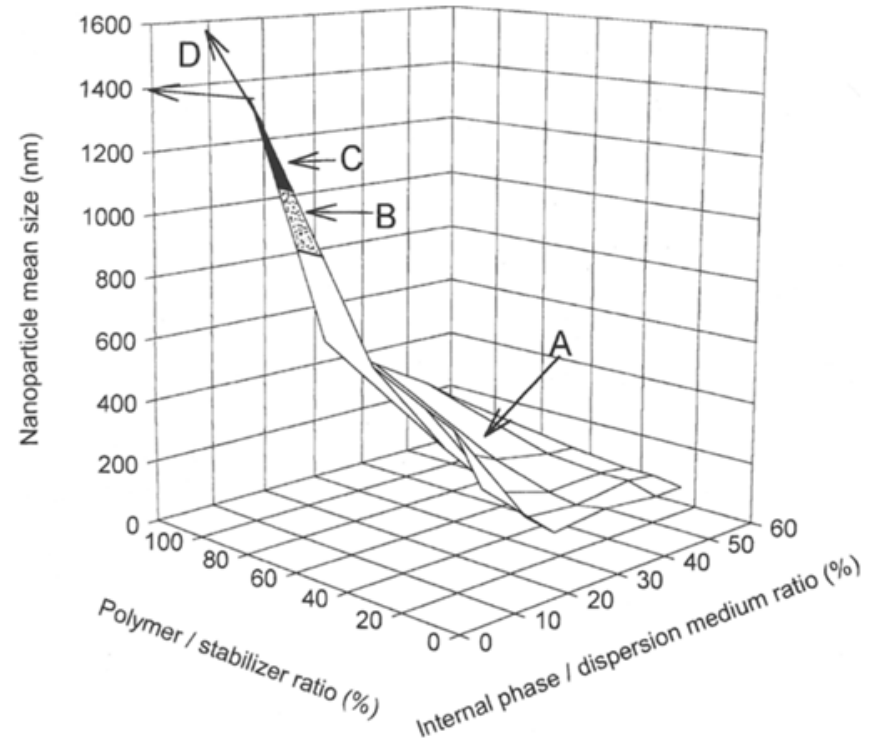

Fig. 3 Effect of PLA/PVAL ratio and internal phase/dispersion medium ratio on the mean size of the nanoparticles. Zone A: nanoparticle formation; Zone B: nanoparticle formation with high polydispersity and presence of polymer flocs; Zone C: microparticle formation with polymer flocs; Zone D: Polymer aggregates or PLA insolubility (30 experimental points) particle per emulsion droplet. Thus, a gradual solvent diffusion of the original globule to the continuous phase and subsequent aggregation of the contained polymer cannot be invoked in the emulsification-diffusion technique.

It is possible that interfacial phenomena during diffusion contribute to the subdivision of the globule into nanodroplets before nanoparticle formation. Previously [6], it has been suggested that the nanodroplets were formed by interfacial turbulence [10] generated during diffusion. This mechanism has been used to explain the formation of nanospheres [11] and nanocapsules [12] by the solvent diffusion technique patented by Fessi et al. [13]. When a polymer solution in acetone is poured or injected into a aqueous stabilizer solution (i.e. poly(vinyl alcohol)), a violent spreading is observed due to the mutual diffusion between the solvents. Droplets of oil, probably of nanometric size, are torn from the interface. These droplets are rapidly stabilized by the stabilizing agent, until diffusion of the solvent is complete and polymer aggregation has occurred. Davies and Rideal [14] have suggested that the interfacial turbulence is caused by localized lowering of the interfacial tension, where the oil phase undergoes rapid and erratic pulsations or "kicks", each of which is quickly damped out by viscous drag. The energy necessary for these jerky movements comes from the free energy released as the solvent is redistributed to its equilibrium state. The molecular mechanism of interfacial turbulence could be explained by the continuous formation of eddies of solvent (e.g. acetone) at the interface. Such eddies may originate either during drop formation, in thermal inequalities in the system, or, once the process has started, in movements associated with previous kicks, that change the pressure inside the solvent either by increasing the surface pressure or decreasing the interfacial tension. Thus, if the solvent droplets formed contain polymer, this will tend to aggregate and form nanoparticles due to the continuous diffusion of solvent and because of the presence of a nonsolvent medium [12]. A schematic representation of these phenomena is shown in Fig. 4. The term nanoprecipitation $[11,15]$ is frequently used to define this process; however, it is important to point out that according to the mechanism described, the formation of nanoparticles is due to polymer aggregation in stabilized emulsion droplets, and the nucleation and growth steps are not apparently involved.

In contrast to the solvent diffusion technique, the partially water miscible solvents used for the emulsification-diffusion technique (PC, benzyl alcohol, etc.) do not emulsify spontaneously, as does acetone. Dimitrova et al. [16] have established that superficial interfacial instabilities can turn out to be only a necessary, but not a sufficient condition for spontaneous emulsification. In other words, even when the interface is unstable, no emulsification will 
be observed if the coalescence rate of the droplets is high enough. Therefore, a great interfacial area (emulsification) must be generated before the diffusion step, to ensure nanoparticle formation. Although the diffusion pattern is different from that observed during the diffusion technique, the interfacial turbulence could explain the subdivi-

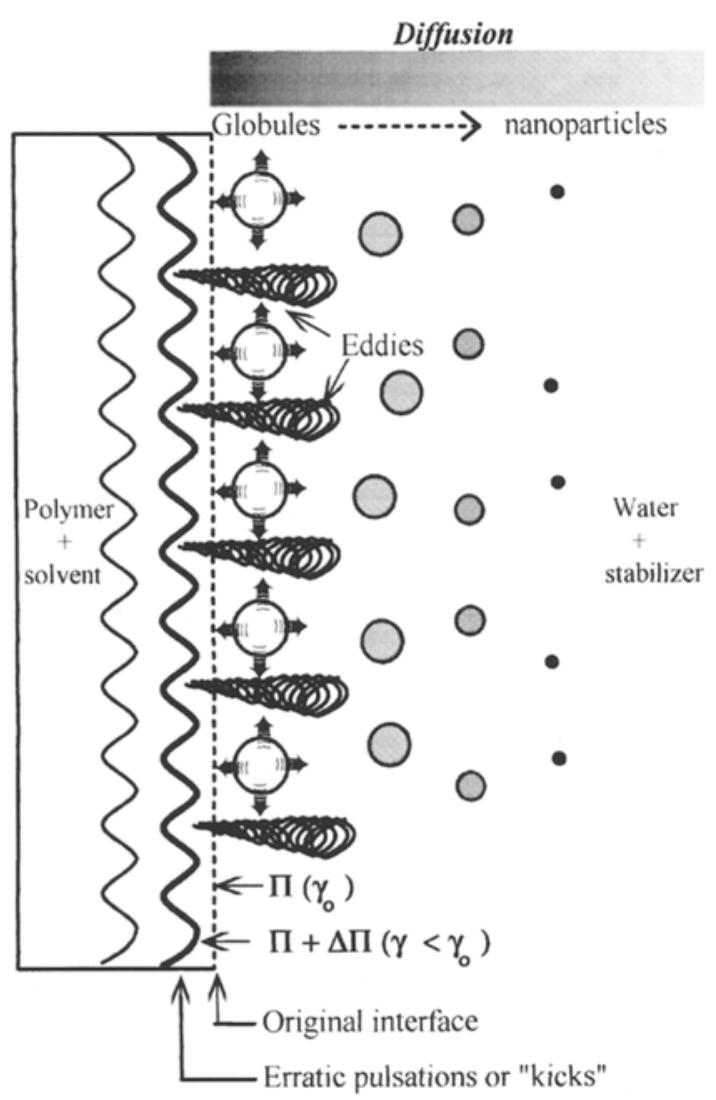

Fig. 4 Schematic representation of nanoparticle formation by the solvent diffusion process based on the interfacial turbulence mechanism, where $\Pi$ and $\gamma$ represent the surface pressure and the interfacial tension, respectively sion of the original globules during the diffusion step. In order to better understand the role of the interfacial turbulence in the emulsification-diffusion technique, some experiments were performed in which the viscosity and the rate of dilution-medium addition were modified. The concentration of PLA in the PC solution $(2 \% \mathrm{w} / \mathrm{v})$, and the ratio of oil/water phases $(1: 2)$ were kept constant. Ideally, if interfacial turbulence is involved, changes of these parameters will affect the diffusion rate of PC and, consequently, the particle size. Tables 1 and 2 show, somewhat unexpectedly, that this seems not to be the case. The division of the original globule from 2600-3200 to $<300 \mathrm{~nm}$ particles, was observed in all cases. A difference of $\sim 100 \mathrm{~nm}$ was obtained between the lowest and the highest viscosity of $175(\eta=1 \mathrm{mPas})$ to $274 \mathrm{~nm}(\eta=$ $277 \mathrm{~m} \mathrm{Pas}$ ). This behavior can be attributed to a probable concentration of the nanodroplets in the interfacial region, where they are able to coalesce and to produce bigger particles. The increase in the polydispersity index seems to support this hypothesis.

Davies and Rideal [14] showed that the interfacial turbulence can be suppressed, at least partially, by dissolving a salt (e.g. $1 \mathrm{~N} \mathrm{NaCl})$ in the dilution medium. The presence of an electrolyte reduces the solubility of the added solvent, thus avoiding the violent interfacial spreading. A further series of experiments was therefore performed by preparing nanoparticles with $1 \mathrm{~N} \mathrm{NaCl}$ in the dispersion and dilution media $(100 \mathrm{ml}$ of the dilution medium were used to ensure the total diffusion of PC). In the interpretation of these experiments, the presence of electrolyte was considered to increase the adsorption properties of PVAL [17].

No difference in particle size was observed between the batches prepared with or without $\mathrm{NaCl}$. These results suggest that interfacial turbulence does not entirely explain nanoparticle formation by the emulsification-diffusion method. On the contrary, batches prepared according to the solvent diffusion technique, by injecting $2 \% \mathrm{w} / \mathrm{v}$ PLA solutions in acetone, into $100 \mathrm{ml}$ of an aqueous
Table 1 Influence of the dilution medium viscosity on the mean size of the nanoparticles

\begin{tabular}{|c|c|c|c|c|}
\hline $\begin{array}{l}\text { Batch } \\
\text { No. }\end{array}$ & $\begin{array}{l}\text { External diffusion phase } \\
\text { viscosity }[\mathrm{m} \mathrm{Pa} \mathrm{s}]\end{array}$ & $\begin{array}{l}\text { Mean size } \pm \mathrm{SD}^{\mathrm{a}} \\
{[\mathrm{nm}]}\end{array}$ & Polydispersity $^{\mathrm{b}}$ & Aggregates $^{c}$ \\
\hline 1 & 1.0 & $175 \pm 6$ & 0 & - \\
\hline 2 & 6.2 & $212 \pm 11$ & 1 & - \\
\hline 3 & 11.7 & $248 \pm 3$ & 1 & - \\
\hline 4 & 23.8 & $242 \pm 7$ & 1 & - \\
\hline 5 & 101.5 & $288 \pm 16$ & 2 & - \\
\hline 6 & 163.0 & $263 \pm 15$ & 3 & - \\
\hline 7 & 276.9 & $274 \pm 13$ & 6 & - \\
\hline
\end{tabular}


Mechanistic study of formation of polymer nanoparticles

Table 2 Influence of the dilution medium addition rate on the mean size of the nanoparticles

\begin{tabular}{lllll}
\hline $\begin{array}{l}\text { Batch } \\
\text { No. }\end{array}$ & $\begin{array}{l}\text { Water addition } \\
\text { rate }[\mathrm{ml} / \mathrm{s}]\end{array}$ & $\begin{array}{l}\text { Mean size } \pm \mathrm{SD}^{\mathrm{a}} \\
{[\mathrm{nm}]}\end{array}$ & Polydispersity $^{\mathrm{b}}$ & Aggregates $^{\mathrm{c}}$ \\
\hline 8 & 0.31 & $188 \pm 3$ & 0 & - \\
9 & 1.01 & $187 \pm 6$ & 1 & - \\
10 & 2.20 & $185 \pm 3$ & 1 & - \\
11 & 6.61 & $167 \pm 4$ & 0 & - \\
12 & 13.32 & $171 \pm 3$ & 0 & - \\
13 & 19.22 & $165 \pm 3$ & 1 & - \\
14 & 26.08 & $169 \pm 3$ & 0 & - \\
\hline
\end{tabular}

a.b.c the abbreviations are the same in Table 1. solution of $5 \% \mathrm{w} / \mathrm{v}$ PVAL in the presence and absence of $1 \mathrm{~N} \mathrm{NaCl}$, revealed the clear influence of partial suppression of the interfacial turbulence. The size increased from $265 \pm 23 \mathrm{~nm}$ (polydispersity index $=2$ ) to $782 \pm$ $93 \mathrm{~nm}$ (polydispersity index $=6$ ) due to the presence of the electrolyte; this means that turbulence plays an important role in the formation of nanoparticles by the solvent diffusion technique.

Therefore, the formation of nanoparticles with the emulsification-diffusion method is attributed principally to solvent transport. In order to explain these phenomena for the spontaneous emulsification processes of ternary systems with mass transfer, Davies and Rideal [14] used a mechanism called "diffusion and stranding", which involves a chemical instability and is entirely different from the interfacial turbulence mechanism which involves a mechanical instability. According to these authors, when an oil-solvent solution, is placed gently in contact with water, the solvent diffuses from the solution into the water carrying with it some oil (forming a three-component phase in the immediate vicinity of the interface). As the solvent diffuses further into the water, the associated oil is thrown out of solution, and is "stranded" in the water in the form of fine emulsion drops. Ruschak and Miller [18] have established that this type of spontaneous emulsification originates in the formation of regions of local supersaturation in one or both phases during the diffusion process, even though, both initial mixtures are unsaturated. It is postulated that such supersaturation cannot persist, and that emulsion droplets form due to phase transformation in these regions. Supersaturation near an interface may also promote its breakup by a distinct but closely related chemical instability mechanism. This "diffusion and stranding" mechanism can be sufficiently extensive to explain the formation of nanoparticles by the emulsification-diffusion technique. The basic idea is that diffusion of PC from the globules carries polymer molecules into the aqueous phase, forming local regions of supersaturation from which, new globules or polymer aggregates (not totally desolvated) are formed; the stabilization of these "protonanoparticles" by the presence of
PVAL is very important in order to avoid their coalescence and the formation of agglomerates. The importance of this stabilization mechanism has been discussed previously [6]. Finally, complete diffusion of PC from the droplet will provoke polymer consolidation in the shape of nanoparticles (Fig. 5). The results of the turbidimetry determinations are consistent with this mechanism. Figure 6 shows the change in $\tau$ during diffusion at different PLA concentrations. The increase in $\tau$ can be attributed to the formation of "protonanoparticles" (increase of the number of particles) and the aggregation of PLA (particles more opaque). For all the PLA concentrations, a gradual increase in $\tau$ is observed, until a limit is reached which is indicative of the final step of "protonanoparticle" formation; then, the decrease in turbidity is explained by the consolidation of the "protonanoparticles", that is the total diffusion of PC and overlap of PLA chains, and by noncorrected dilution effects. It is important to mention that the point at which $\tau$ begins to increase and the time at which the maximum value is reached, are PLA concentrationdependent. For PLA concentrations of $5 \%$ and $7.5 \% \mathrm{w} / \mathrm{v}$, nanoparticle formation begins as the first volume of the diffusion medium is added, while for PLA concentrations of $2 \% \mathrm{w} / \mathrm{v}$ and less, $\tau$ decreases until a certain limit is reached; this fact could suggest that $\mathrm{PC}$ diffuses without carrying PLA, until a polymer concentration in the globules is reached where protonanoparticles begin to appear. This behavior could explain the similarity in particle size (Fig. 3) found with PLA concentrations lower than $5 \% \mathrm{w} / \mathrm{v}$. On the other hand, with PLA concentrations greater than $5 \% \mathrm{w} / \mathrm{v}$, the size gradually increases with the consequence that the "protonanoparticles" generated during diffusion are more abundant and are highly concentrated at the interface. The probability that they can collide and coalesce among themselves, or with the interface, is thus greater than at low concentrations. Figure 7 shows the influence of stirring on $\tau$ for batches prepared at the same PLA concentration $(2 \% \mathrm{w} / \mathrm{v})$. The peak intensity increased with stirring rate and was inversely proportional to the particle size obtained (Table 3 ), so we can say that the globule size of the original emulsion determines, to 
Fig. 5 Schematic description of the proposed formation mechanism of PLA nanoparticles by the emulsification-diffusion method based in the diffusion stranding mechanism

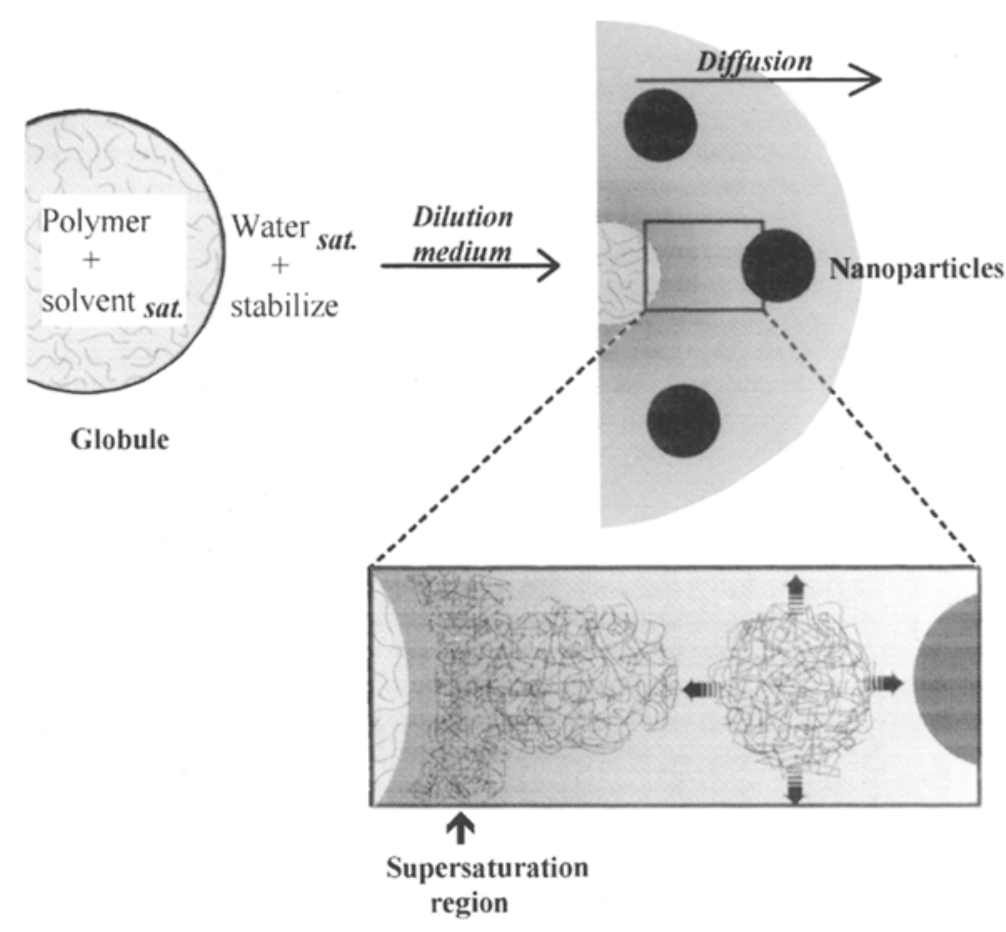

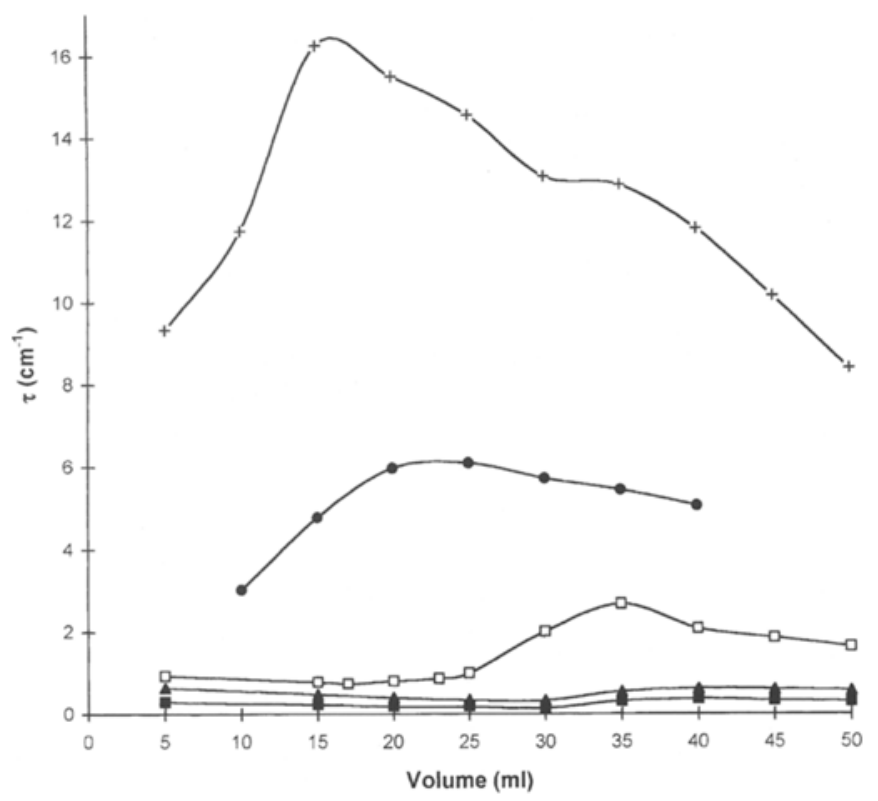

Fig. 6 Evaluation of the turbidimetric changes during the addition of dilution medium for batches prepared with different concentra-

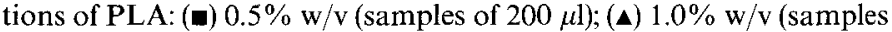

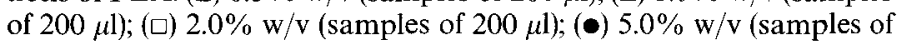
$100 \mu \mathrm{l}) ;(+) 7.5 \% \mathrm{w} / \mathrm{v}($ samples of $50 \mu \mathrm{l})$

a certain extent, the particle size. This fact could be explained by the difference in thickness of the supersaturated region and the rapid formation and stabilization of protonanoparticles at high stirring rates. For coarse emulsions

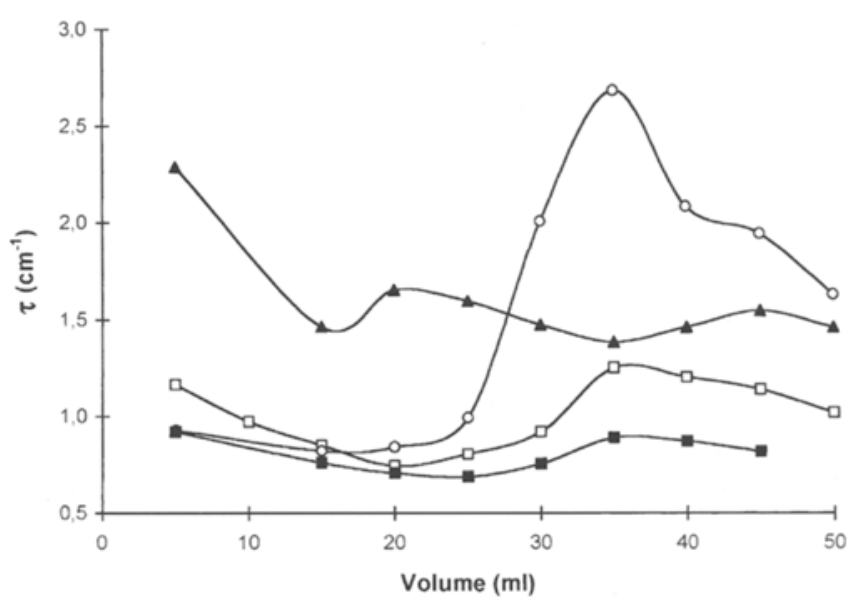

Fig. 7 Evaluation of the turbidimetric changes during the addition of dilution medium for batches prepared at different stirring rates: $(\Delta)$ $500 \mathrm{rpm}$; (ロ) $1500 \mathrm{rpm}$; $\square 2000 \mathrm{rpm}$; (O) $9000 \mathrm{rpm}$ (samples of $200 \mu \mathrm{l}$ )

(500 rpm), the curve showed a very different behavior; the large globule size and the probable coalescence could provoke the formation of a polymer film around the globules, but the polymer concentration is insufficient to obtain supersaturated regions and therefore nanoparticle formation is not observed. Experiments with droplets of $50 \mu 1$ of PLA $2 \% \mathrm{w} / \mathrm{v}$ solution, injected into PVAL solutions, showed this behavior. These results suggest that the exposed superficial area generated during emulsification is an important factor for the superficial phenomena 
Table 3 Influence of the stirring rate on the mean size of the nanoparticles

\begin{tabular}{lllll}
\hline $\begin{array}{l}\text { Batch } \\
\text { No. }\end{array}$ & $\begin{array}{l}\text { Stirring rate } \\
{[\mathrm{rpm}]}\end{array}$ & $\begin{array}{l}\text { Mean size } \pm \mathrm{SD}^{\mathrm{a}} \\
{[\mathrm{nm}]}\end{array}$ & Polydispersity $^{\mathrm{b}}$ & Aggregates $^{\mathrm{c}}$ \\
\hline 15 & 500 & $1660 \pm 158$ & 7 & + \\
16 & 1500 & $242 \pm 14$ & 3 & - \\
17 & 2000 & $212 \pm 6$ & 1 & - \\
18 & 9000 & $171 \pm 3$ & 0 & - \\
\hline
\end{tabular}

a.b.c the abbreviations are the same as in Table 1. involved in the diffusion step and for controling the particle size obtained.

\section{Conclusions}

The present work has shown that nanoparticle formation by the emulsification-diffusion process involves interfacial phenomena during the diffusion step that contribute to the generation of colloidal particles. The convection due to interfacial turbulence apparently does not influence nanoparticle formation. The results support the hypothesis that solvent diffusion produces regions of local supersaturation from which, nanoparticles are formed, due to phase transformation in these regions. Preparative vari- ables such as polymer concentration and stirring rate could be important factors for the formation and thickness of the supersaturated region, and might change the mean nanoparticle size. We point out that the mechanism of nanoparticle formation with the emulsification-diffusion process proposed in this paper is not attributed to a mechanical instability, but to a chemical instability and is closely related to that for isolated precipitation in solid systems.

Acknowledgments D.Q.G. acknowledges a grant from CONACYT and FES-Cuautitlan, UNAM, México. The authors are grateful to Mrs. Danielle Massuelle for technical assistance with the scanning electron microscope and Dr. Yogeshvar N. Kalia for critically reviewing the manuscript.

\section{References}

1. Allémann E, Gurny R, Doelker E (1993) Eur J Pharm Biopharm 39:173-191

2. Couvreur P, Dubernet C, Puisieux F (1995) Eur J Pharm Biopharm 41:2-13

3. Gurny R (1983) In: Breimer DD, Speiser $P$ (eds) Topics in Pharmaceutical Sciences. Elsevier, Amsterdam, pp 277-288

4. Gallardo MM, Roblot-Treupel L, Mahuteau J, Genin I, Couvreur P, Plat M, Puisieux F (1989) Nanocapsules et nanosphères d'alkyl-cyanoacrylate, interactions principe actif/polymère. Proc APGI, 5th Int Conf on Pharmaceutical Technology, Paris (France) pp 36-45

5. Leroux JC, Allémann E, Doelker E, Gurny R (1995) Eur J Pharm Biopharm 41:14-18
6. Quintanar-Guerrero D, Allémann E, Fessi H, Doelker E (1996) Int J Pharm 143:133-141

7. Sánchez A, Vila-Jato JL, Alonso MJ (1993) Int J Pharm 99:263-273

8. Elving PJ, Winefordner JD (1982) In: Chemical Analysis. Wiley, New York, pp 255-257

9. El-Aasser $M$ (1979) In: Advances in Emulsion Polymerization and Latex Technology. Vol 2. Lehigh University, Bethleem, Pennsylvania, No. 19

10. Sterning CV, Scriven LE (1959) AIChEJ 5:514-523

11. Molpeceres J, Guzman M, Aberturas MR, Chacon M, Berges L (1996) J Pharm Sci 85:206-213
12. Fessi H, Puisieux F, Devissaguet JP, Ammoury N, Benita S (1989) Int J Pharm 55: R1-R4

13. Fessi H, Devissaguet JP, Puisieux F, Thies C (1988) French Pat 2608988

14. Davies JT, Rideal EK (1961) In: Interfacial Phenomena. Academic Press, New York, pp 319-326, pp 359-366

15. Stainmesse S, Orecchioni AM, Nakache E, Puisieux F, Fessi H (1995) Colloid Polym Sci 273:505-511

16. Dimitrova B, Ivanov $Y B$, Nakache $E$ (1988) Disp Sci Tech 9:321-341

17. Tadros TF (1979) J Colloid Interface Sci $72: 505-514$

18. Ruschak KJ, Miller CA (1972) Ind Eng Chem Fundam 11:534-540 